\title{
HyPER-REALISM IN THE ADVENTURES OF TINTIN
}

\author{
Chris Carter \\ Animation, Queensland University of Technology, Brisbane, Australia
}

\begin{abstract}
The Adventures of Tintin: The Secret of the Unicorn is an animated feature film that blends graphic stylisation with photorealistic surfaces and motion capture to create digital beings in a hyper-realistic style. As a result, the visual style of The Adventures of Tintin is significantly different to the now dominant cartoon stylisation that continues to develop in studios such as Disney, PIXAR and many others. Disney inspired cartoon stylised films, such as Frozen, continue to engage audiences around the world; receiving relatively little criticism for their visual style. Hyper-realistic animation, however, has struggled to achieve the same widespread acceptance. Many people continue to find hyper-realistic characters less engaging than cartoon characters and at times even find them to be somewhat repulsive. Via close analysis of The Adventures of Tintin, this paper illuminates the significance of establishing visual harmony between the elements of shape, surface and motion to sustain appealing and believable hyper-realistic characters.
\end{abstract}

\section{KEYWORDS}

Animation, Hyper-realistic, Tintin, 3D Animation, Animation Aesthetics, Character Animation

\section{INTRODUCTION}

Films such as Final Fantasy: the Spirits Within[1], The Polar Express[2], Beowulf[3], A Christmas Carol[4] and Mars Needs Moms[5] have all under-performed at the box office and received widespread criticism for featuring characters that lack believability. The connection of appearance and behaviour, modelling of complexity and the interaction of the digital character are three fundamental problems that Flückiger[6] associates with the construction of digital beings. While all three problems are important, it is the connection of appearance and behaviour that is of particular interest to this paper. A digital character's appearance and behaviour is made visible to the audience via three interconnected elements that include the character's shape, surface and motion. When handled in a certain manner, the dynamic interaction of these elements can create the illusion of a living, thinking being capable of forming and sustaining an empathetic bond with an audience. When these elements become unstable or dissonant, however, the believability of the digital being is diminished and the illusion of life is threatened. The interaction between the elements of shape, surface and motion provides a point of entry for theorists and practitioners to establish principles that may guide the creation of believable digital beings.

\section{ANALYSIS}

Creating a believable digital being has continued to challenge artists and technicians for some time, consequently, several approaches to their evaluation have begun to emerge. Perhaps the most widely acknowledge model for evaluating digital beings is The Uncanny Valley theory, which originated from the field of android science by roboticist Masahiro Mori [7].

DOI: $10.5121 /$ ijcga.2019.9401 
Although primarily based on conjecture, Mori described a phenomenon in which "perceived familiarity increases with human likeness until a point at which subtle deviations from human appearance and behaviour create an unnerving effect" [8, p. 299]. The unnerving effect Mori encountered was what psychologist Ernst Jentsch[9, p. 1] referred to as "the affective excitement of the uncanny". A simplified figure of Mori's Uncanny Valley theory is shown below:

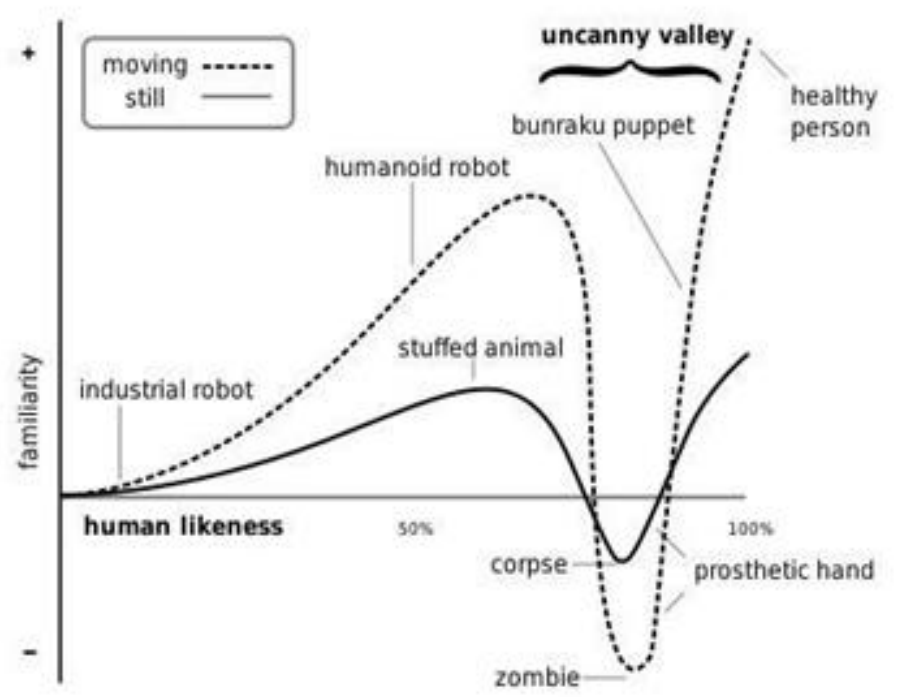

Figure 1: Simplified Figure of Mori’s Uncanny Valley [8, p. 299].

As the figure above shows, Mori posited that human familiarity increased as the appearance of an object moved toward human likeness until a point at which familiarity would sharply decline and arouse an uncanny response in the person. As indicated by the dotted line, Mori also observed an amplification of the uncanny effect when movement was added. Although appearing photorealistic on the surface, characters may appear to lack qualities made visible through motion introduced to create the illusion of life, such as a sense of weight, physicality and breath.

This was apparent in the film Final Fantasy: The Spirits Within[1], which has become widely recognised as an exemplar of the Uncanny Valley in computer animated films. Flückiger explains that although "photorealistic on the surface, these characters lack various human traits. They seem not to breath and they have a very limited expressive repertoire. In short, they seem somehow dead." [6, p. 41]. As Mori's Uncanny Valley theory suggests, the living dead or zombie as it appears on Mori's graph, is where human familiarity is at its lowest and therefore the bottom of the valley.

Despite Mori's Uncanny Valley conjecture being widely acknowledged and even permeating popular culture, theorists such as Flückiger[6], Hanson [10] and Tinwell[11] have recognised that the uncanny valley conjecture is inadequate for understanding the full aesthetic range of modern digital beings or androids. Flückiger, offers the case of Gollum from The Lord of Rings[12]-[14] as justification for "seeking alternative explanatory models" [6, p. 42]. She argues that Gollum is a successful example of a digital being that does not fit the Uncanny Valley model because he diverges in some respects from his seemingly human qualities [6, p. 42]. More recently, other 
digital beings have continued to challenge the Uncanny Valley theory as new techniques and aesthetic possibilities are explored. The characters in The Adventures of Tintin[15]for example are difficult to place along Mori's path of familiarity because of the integration of human motion with cartoon stylised proportions and hyper-realistic textures.

The need for a revised theory is therefore amplified with cases such as The Adventures of Tintin, which move beyond photorealism toward a stylised version of reality or what VFX supervisor Matt Aitken referred to as an "idealised reality" [16]. The characters are shaped like exaggerated cartoon figures that move like natural humans and their surface textures are almost photorealistic. Are they a cartoon or an attempt to simulate a real person? This question prevents them from finding a position on either side or Mori's uncanny valley because they are neither realistic or cartoon. Unlike other films, such as The Polar Express[2] or Mars Needs Moms[5], the characters in Tintin(for the most part)manage not to fall into the valley either. Situations such as this, are now causing people to challenge Mori's conjecture further.

For example, David Hanson [10] from Hanson Robotics goes as far to argue that the "valley" does not even exist. As an alternative to Mori's Uncanny Valley, Hanson has proposed a paradigm he labels the Path of Engagement (POE):

If the illusion of life can be created and maintained, the uncanny effects may be mitigated. It may be that any level of realism can be socially engaging if one designs the aesthetic well. This, in effect, would represent a bridge of good aesthetic, which inspires us to name the revised theory the path of engagement (POE). [17, p. 4]

As Hanson's Path of Engagement theory suggests, perhaps an engaging aesthetic is all that is needed to sustain the illusion of life at any level of realism. This is consistent with the findings from an empirical study by Flückiger[6], which involved the close analysis of digital beings in Hollywood feature films.

Rather than rebut the existence of the uncanny valley affect, Flückiger tries to provide an alternative model that can account for a wider range of aesthetic and behavioural characteristics of digital beings. Flückiger proposes an approach she describes as the "model of distance" [6, p. 42], which measures the deviation of the appearance and behaviour of a character from a defined standard value. She explains:My hypothesis is that the different aspects of a character should be situated at a similar distance from a transparent mode of representation. Between photorealistic and stylized depictions there runs a fine yet essential line, which separates fundamentally different forms of representation. [6, p. 42]

Unlike the Uncanny Valley theory, Flückiger's distance model proves to be a robust approach to analysing the characters in Tintin. The "essential line" indicated by Flückiger is of particular interest as it represents the critical point at which abstract forms approach photo realism. The matrix below shows the various aspects of characters in Tintinplotted in relation to the parameters specified by Flückiger: 


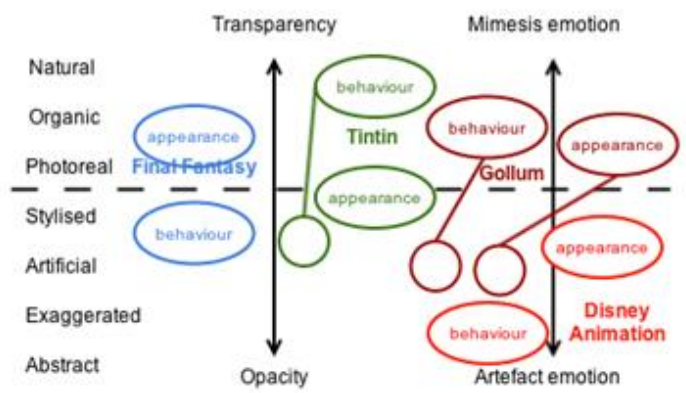

Figure 2: Tintin shown against Flückiger's distance model.

All the fundamental elements of a digital being (shape, surface, motion) can be "projected" onto this matrix. For the benefit of comparison, Final Fantasy, Gollum and Disney Animation appear in my reproduction of the diagram as Flückiger originally mapped them. In Flückiger's own words, the matrix:

..depicts the various parameters, arranged on the left side in a continuum spanning the natural to the abstract. At one end there are hypothetical, transparent forms of representation, which afford an undistorted view of the depicted object world, comparable to looking out of a window, and thereby evoke emotions through mimesis. At the other end there are opaque forms of representation that guide attention toward their own materiality and/or virtuosity, and thereby evoke artifact emotions. [8, p. 42, 43]

As previously mentioned, Final Fantasy is well recognised as a failed attempt at creating believable digital beings. As Flückiger has shown via the distance model, the characters in Final Fantasy fail, because appearance and behaviour are situated on opposing sides of the essential line. In comparison, as I have shown in the above diagram, the characters in Tintin are not entirely successful because various aspects of the characters appearance and behaviour cross the essential line.

More specifically, the character appearance in particular, in Tintin straddles the essential line by mixing stylised proportions with photo real surfaces. Matt Aitken explains the visual style of the characters:

Getting the look of the characters right was one of the big challenges of this production. Right from the start we wanted to honor the design that Hergé created for his characters, that signature look. But our characters also had to believably exist in the world we were creating for them: a highly detailed world of idealized reality. We used exaggerated facial features and the proportions of the comic book characters with their oversized heads and hands to carry the look of Hergé's characters on into the world of the film. But we made the details of the characters natural: their skin textures, the way their faces moved when they spoke and especially their eyes are much closer to real humans than their overall form. [16]

This approach places the character at risk of failing to sustain believability because the stylised character proportions and shape create a level of visual dissonance in the spectator. Visual dissonance is closely related to the concept of cognitive dissonance from the field of social psychology, Solso[18, p. 235] describes visual dissonance as "a state of psychological tension 
caused when one experiences a disparity between what one expects to see and what one actually sees". In Final Fantasy, the visual dissonance caused by the characters could be considered a byproduct of technological limitations, which resulted in poor movement quality and an insufficient level of visual complexity that was unable fool the audience into thinking the characters were completely real. In Tintinhowever, visual dissonance is caused by the disparity of the cartoon shape and the surface detail; spectators are not accustomed to and therefore do not expect to see a cartoon shaped human with realistic surface textures. Adding to the level of visual dissonance is the characters movement and behaviour.

The character shape in Tintin suggests to the audience that these are cartoon characters, therefore audiences anticipate stylised movement. However, movement in Tintin was created, for the most part, via performance capture, which is a process that involves recording and mapping human motion onto the virtual figure. The result is very naturalistic motion that doesn't quite fit the exaggerated proportions of the digital character, whose head and hands at times appear as though they are being moved more like a marionette than from their own internal forces.

This breakdown in the illusion of life when copying motion from live action reference onto stylised cartoon figures is not limited to $3 \mathrm{D}$ computer animation, the process has always proved to be a challenge, as Disney animators Thomas and Johnston explain of the rotoscope:

There was a certain authority in the movement and a presence that came out of the whole action, but it was impossible to become emotionally involved with this eerie, shadowy creature who was never a real inhabitant of our fantasy world. [19, p. 323]

This "eerie" effect is minimised in Tintin somewhat, as captured motion data has been modified by animators to make it more appealing. Spielberg described it as being able to "underwrite or overwrite a performance and through the animators put [something into a performance] that even the actors didn't bring to the bay" [20]. As a result, the character motion at times appears more stylised by becoming slightly more exaggerated and even breaking the laws of physics. This is represented on the matrix above by the satellite-like extension that crosses the essential line and brings an element of balance to the character's appearance and behaviour. Character appeal has long been understood by animators as fundamental to creating believable characters.

Disney animators, Frank Thomas and Ollie Johnston went as far as to list appeal as one of the 12 fundamental principles of character animation that were identified at the Disney studio during the 1930s [19]. The principle of appeal, as it pertains to character animation, is a concept encompassing all aspects of the character including its appearance and behaviour. That is to say appeal is concerned with "anything that a person likes to see, a quality of charm, pleasing design, simplicity, communication, and magnetism" [19, p. 68]. Aesthetic appeal is subjective, however, through continuous refinement, Disney animators developed a cartoon style that was appealing to a large and diverse audience. This applies to characters of all forms such as villains and monsters and not just "cuddly bunnies and soft kittens" [19, p. 68]. At a basic level, Disney characters are simplified graphic representations of people and anthropomorphised animals or objects constructed from simple shapes with minimal surface detail. An appealing design captures the audience's attention, contributes to the building of character and promotes audience identification with the situation represented in the story $[19$, p. 68]

In Understanding Comics[21], Scott McCloud explains that audience involvement is highly dependant on "the degree to which the audience identifies with a stories characters" [21, p. 42]. 
According to McCloud, the contrast of simplified, iconic character forms placed against detailed backgrounds promotes viewer identification, as he explains in the image below:

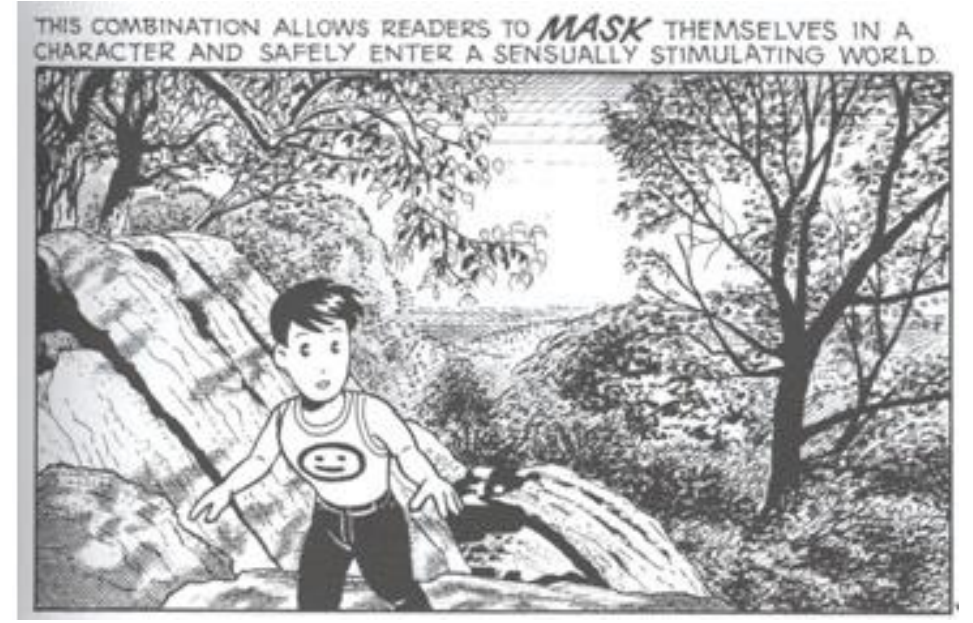

Figure 3: Simplified character against a detailed background [21, p. 43].

Although McCloud is specifically referring to traditional hand drawn animation, this reductive/iconic approach to character design has proven effective in the $3 \mathrm{D}$ computer graphics medium as well. The characters in the 3D animated feature film Up[22] for example are graphic cartoon forms that exist in a highly detailed world, as the image below shows:

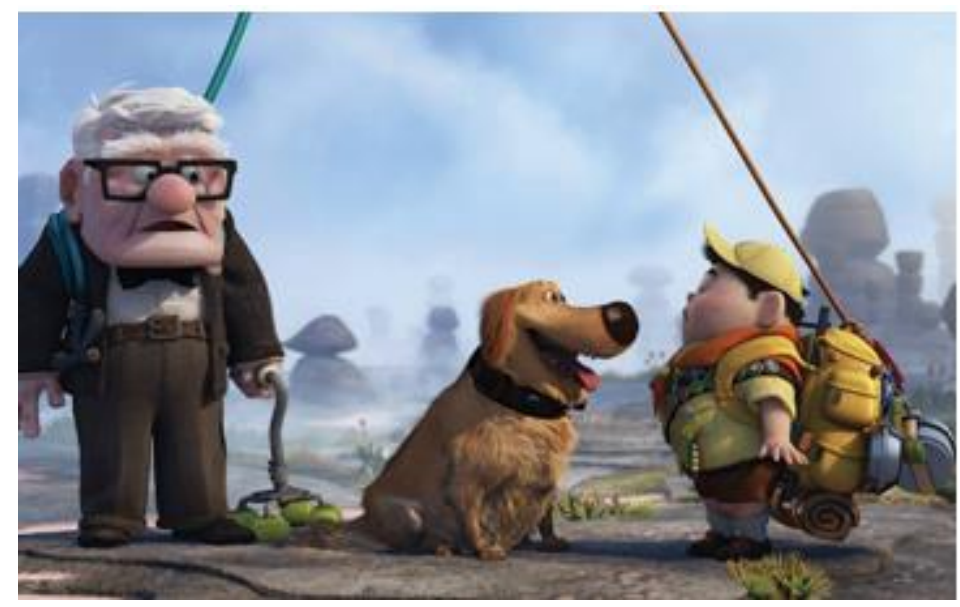

Figure 4: Characters in Up.

When designing the aesthetic for Tintin, Spielberg chose not to follow the Disney approach. Instead, he opted to exaggerate the proportions of the characters in a cartoon style but render their surfaces with the same level of detail as the environment in which they exist. This places pressure on the ability for audiences to identify with the characters as the "masking" effect McCloud describes is diminished. The image below shows a close up of the surface details on the faces of Tintin and Haddock. In comparison to the example above from Up, notethere is a disparity between the exaggerated proportions and detailed surfaces in Tintin: 


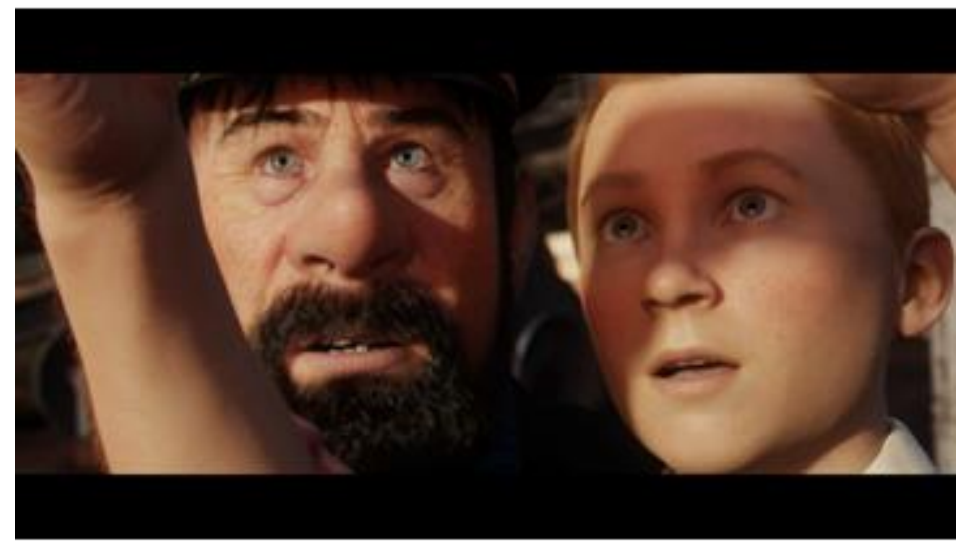

Figure 5: Detailed surfaces of characters in Tintin.

If Solso's[18] explanation of visual dissonance is correct, then an expectation for how Tintin should appear could contribute to a breakdown in the overall appeal of the characters. Georges Prosper Remi's (Hergé) "LigneClarie" art style is well known for combining simplified iconic characters with unusually realistic backgrounds [21, p. 42]. This creates a certain level of expectation amongst viewers of how Tintin may appear as a three dimensional object. One example of this can be found in the existing figurines that represent the iconic characters in a physical three dimensional form
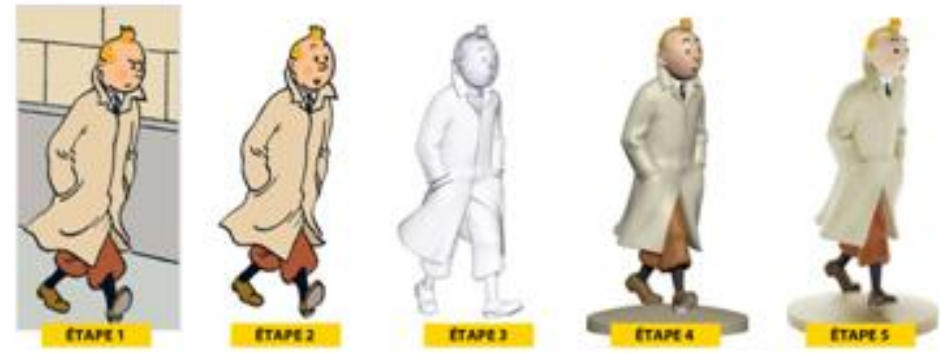

Figure 6: A Tintin figurine developed from and reflecting original art style .

The image above has been sourced from a producer of Tintin figurines. The sequence shows the process of transferring the original hand drawn figure into a virtual 3D object before being printed as a physical model. This simplified form is more closely aligned to the Disney/PIXAR aesthetic and clearly demonstrates that the characters can be represented in 3D yet remain closely aligned to the expectations of Tintin's appearance. The visual style in Spielberg's version of Tintin was therefore no accident, nor was it a result of technological limitations or budget restrictions. The character appearance was a deliberate creative decision that was driven by more than trying to stay true to the original art style.

As the pre-production image below shows, earlier iterations of Tintin's design did actually reflect the iconic design of Hergé more faithfully. However, according to the Weta Digital Models Supervisor, Marco Revelant[15], the expressive range of Tintin's simplified shape was insufficient for handling the range of expressions required of a realistic film. 


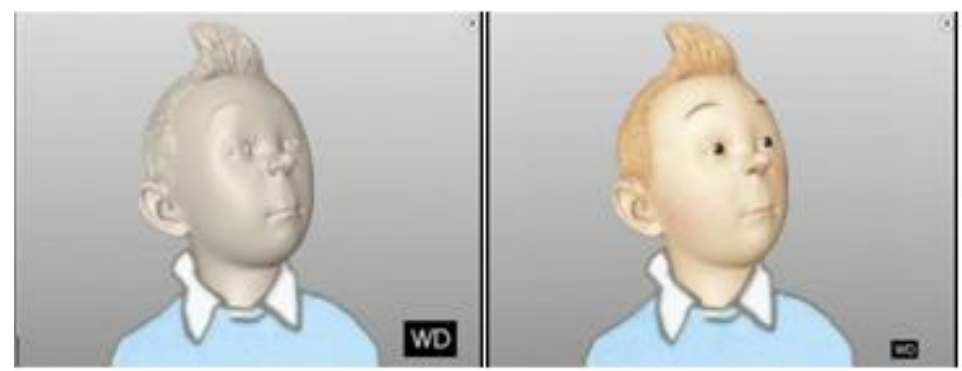

Figure 7: Early concept for translating Tintin to a 3D digital model [15].

As mentioned previously, the modelling of complexity was found by Flückiger to be a fundamental challenge for the creation of believable digital beings. For Tintin the challenge was to create a character with the complexity necessary for representing the full expressive range of human emotion while remaining closely aligned to the simplified graphic stylisation of a flat cartoon character. The final Tintin character design seen in the film was the result of combining the round face and head of classic Tintin with a three-dimensional scan of actor Jamie Bell's head, who's facial expressions would ultimately be mapped onto the digital model [15].

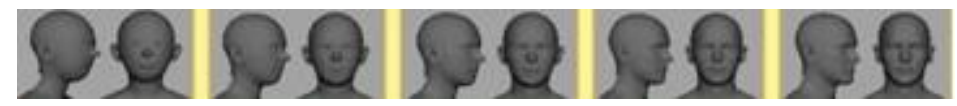

Figure 8: Combining the Tintin model with a 3D digital scan of the actor's head [15].

Balancing the shape of the character in this way could explain why the characters in Tintin do not fail to the same degree as other films that cross over to the realistic side of Flückiger's essential line. According to Solso[18, p. 235], there are three possible ways to reduce visual dissonance: (1) reducing the importance of one of the dissonant elements, (2) reinterpreting one or more of the elements, or (3)

changing one of the dissonant elements. In Tintin, the importance of both the cartoon stylisation and the level of photorealism has been reduced, which has resulted in a more balanced aesthetic than other attempts at hyper-realistic 3D CG characters.

Perhaps the characters in Tintin could have been even more successful had the elements of shape, surface and motion been more uniformly situated on the essential line indicated in Flückiger's model of distance. This is represented in the matrix below as a proposed model of distance for Tintin: 


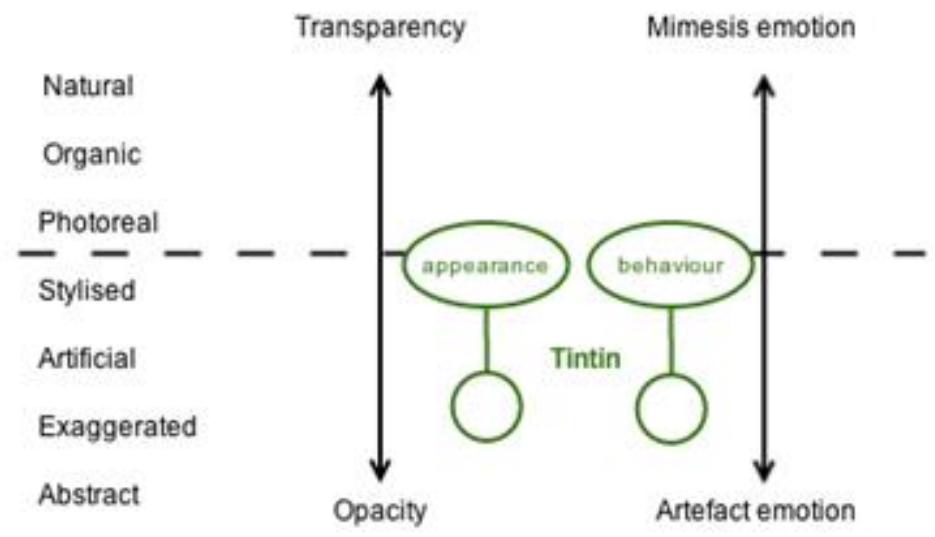

Figure 9: A proposed model of distance for Tintin.

The nodes extending beyond the essential line and toward the abstract end of the spectrum indicate a possible level of abstraction that could be necessary to deal with insufficiently complex modelling, deformation and motion due to technological limitations at the time of production. In addition to the elements of shape, surface and motion operating at a similar distance to a transparent mode of representation, there is also the need to consider the behaviour of that character and how their actions fit within the context of the film.

According to Leslie Bishko[23], authenticity is a determining factor for the believability of a character. She explains that in order to be authentic and therefore believable a character must adhere to at least three criteria, which include:

In the context of craft, congruence of design with expression: when line, shape, form, color, composition, voice acting and movement are aligned with content, forming a fluid and meaningful whole. In the context of characterization, congruence between a character's intent and its resulting action. Alignment of style with content, as opposed to adoption of style without connecting it to its subject. [23]

The characters in Tintin fail to appear authentic when measured against Bishko's criteria. In the context of craft, there is a disparity between the design and expression of the characters and there is a misalignment of style with content. As an animated film with stylised characters, audiences may anticipate a certain level of broad cartoon style action. As my previous publications have shown [24], it is typical in animated films to find subdued movements for dramatic scenes contrast with broad cartoon action for comedy and action scenes. However, as previously mentioned, Spielberg chose to use performance capture for the character movement in Tintin, which places the resulting motion and ultimately the character behaviour at odds with their exaggerated cartoonish proportions.

For example, the images below are taken from a short comedic sequence in Tintin; despite the cartoon like comedy and fast action, the character's body does not deform in a typical squash and stretch cartoon fashion and there is no visible successive breaking of the joints: 

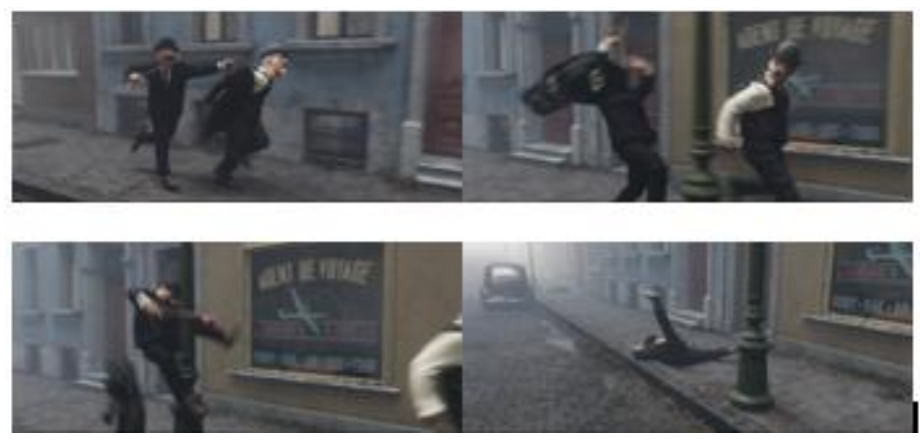

Figure 10: Cartoon action without cartoon motion in Tintin.

Squash and stretch and successive breaking of the joints are two devices developed in the 2D medium to improve the illusion of weight, physicality and flexibility in the characters [25]. As seen in the example above, an absence of these two techniques results in a physicality that appears stiff and constrained to the typical shape and proportions of the stationary character. In this case the character's action seems authentic, however, the motion is at odds with the action and the cartoon proportions of the character.

In a separate example, the character behaviour and motion are both misaligned to the context of the scene. The images below, for example, show Tintin and Captain Haddock after crashing an aeroplane in the desert:
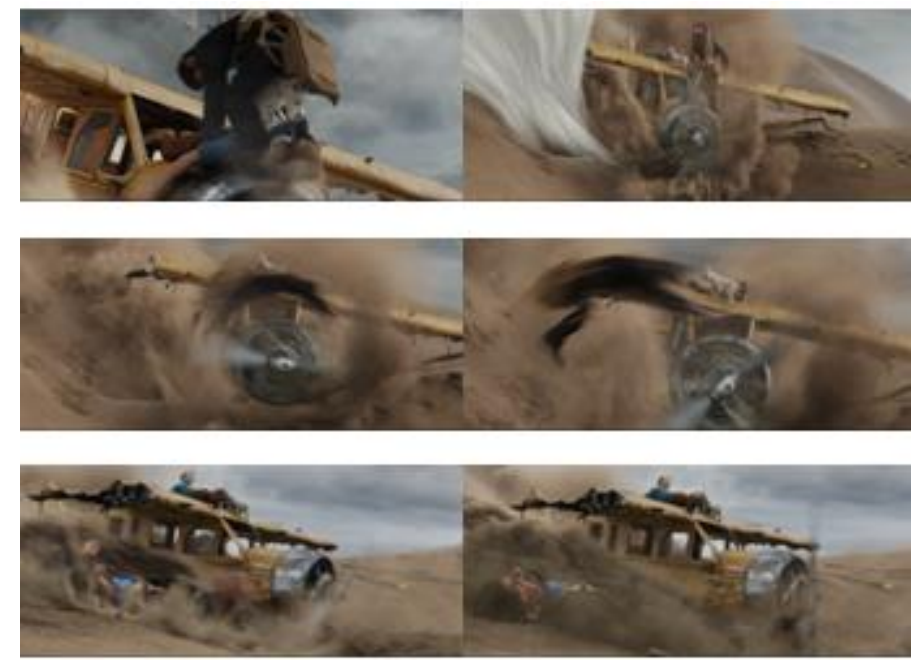

Figure 11: Cartoon action without cartoon motion in Tintin.

The context of this particular scene indicates that Tintin is at risk of being killed if he were to slide into the spinning propeller of the crashed aeroplane. His dog Snowy and Captain Haddock act desperately to prevent Tintin from sliding into the propeller. This establishes in the audience, an expectation that the moving blades of a propeller can fatally injure these characters. However, despite this indication of apparent danger, Haddock is not injured when he himself becomes entangled in the propeller. Instead, Haddock is wound up and flung around like a typical cartoon character. A situation like this would be well suited to a Looney Tunes cartoon or a PIXAR/Disney cartoon style film. However, an event such as this lacks authenticity in a hyper- 
International Journal of Computer Graphics \& Animation (IJCGA) Vol.9, No.4, October 2019

realistic film because the naturalistic motion and actions of the character do not marry well with the sudden cartoon style gag that is shown with no change to the otherwise stiff naturalistic motion.

\section{CONCLuSions}

The Adventures of Tintin successfully challenges the now dominant PIXAR/Disney 3D CG aesthetic to explore a style of animation that blends exaggerated cartoon proportions with naturalistic motion and photorealistic surface textures. This blended aesthetic provides an opportunity to examine how the interaction of the basic elements of shape, surface and motion contribute to (or diminish) the believability of the characters. Existing popular models for evaluating animated characters, such as the 12 principles of animation or the uncanny valley theory, prove to be inadequate for evaluating this visual style. This is because the animation style does not appear to adhere to classic Disney animation principles or attempt to achieve a state of complete photorealism. However, as I have shown in this paper, Flückiger's distance model is able to provide a framework for discussing the interaction of the elements and how they function to connect the characters appearance with behaviour. This approach has shown that when the elements of shape, surface and motion fall out of harmony, the illusion of life is diminished and the believability of the character threatened. Within the context of character animation, visual dissonance gives way to a state of visual harmony when the elements of shape, surface and motion function together to create a character that appeals to a wide audience. If the shape, surface and motion of the characters in Tintin had been more uniformly situated at the same point along the continuum spanning from natural to abstract, the level of visual dissonance could have been minimised and the character's appeal improved. A more stable connection of the visual style with the subject could have further improved the character believability by decreasing the level of visual dissonance that is created by a disparity between the cartoon action and naturalistic motion.

\section{REFERENCES}

[1] S. Hironobu and S. Motonori, Final Fantasy: The Spirits Within. Columbia Pictures, 2001.

[2] R. Zemeckis, The Polar Express. 2004.

[3] R. Zemeckis, Beowulf. Paramount Pictures, 2007.

[4] R. Zemeckis, A Christmas Carol. Walt Disney Studios Motion Pictures, 2009.

[5] S. Wells, Mars Needs Mums. Walt Disney Pictures, 2011.

[6] B. Flückiger, Visual Effects-Filmbilderausdem Computer, vol. 18. Schüren, 2008.

[7] M. Mori, "Bukimi no tani [the uncanny valley]," Energy, vol. 7, pp. 33-35, 1970.

[8] K. F. MacDorman and H. Ishiguro, "The uncanny advantage of using androids in cognitive and social science research,” Interact. Stud., vol. 7, no. 3, pp. 297-337, 2006.

[9] E. Jentsch, “On the Psychology of the Uncanny (1906)," Angelaki J. Theor. Humanit., vol. 2, no. 1, pp. 7-16, 1997.

[10] D. Hanson et al., "Upending the uncanny valley," in AAAI, 2005, vol. 5, pp. 1728-1729. 
International Journal of Computer Graphics \& Animation (IJCGA) Vol.9, No.4, October 2019

[11] A. Tinwell and M. Grimshaw, "Bridging the uncanny: an impossible traverse?," in Proceedings of the 13th International MindTrek Conference: Everyday Life in the Ubiquitous Era, 2009, pp. 66-73.

[12] P. Jackson, The Lord of the Rings: The Fellowship of the Ring. New Line Cinema, 2001.

[13] The Lord of the Rings: The Two Towers. New Line Cinema, 2003.

[14] The Lord of the Rings: The Return of the King. New Line Cinema, 2002.

[15] Weta, The Art of the Adventures of Tintin. New York, NY: Harper Design, 2011.

[16] "THE ADVENTURES OF TINTIN: Matt Aitken - VFX Supervisor - Weta Digital - The Art of VFXThe Art of VFX." [Online]. Available: https://www.artofvfx.com/the-adventures-of-tintin-mattaitken-superviseur-vfX-weta-digital/. [Accessed: 02-Oct-2019].

[17] D. Hanson, "Exploring the aesthetic range for humanoid robots," in Proceedings of the ICCS/CogSci2006 long symposium: Toward social mechanisms of android science, 2006, pp. 39-42.

[18] R. L. Solso, "The psychology of art and the evolution of the conscious brain,” 2003.

[19] F. Thomas and O. Johnston, The illusion of life: Disney animation. Hyperion, 1995.

[20] G. Boucher, "Tintin': Steven Spielberg Says 'the Medium Isn't the Message',," Hero Complex movies, comics, pop culture - Los Angeles Times. .

[21] S. McCloud, Understanding Comics: The Invisible Art (Reprint edition.). New York: William Morrow Paperbacks, 1994.

[22] P. Dpctpr and B. Peterson, Up. Walt Disney Studios Motion Picture, 2009.

[23] L. Bishko, “The uses and abuses of cartoon style in animation,” Animation, vol. 2, pp. 24-35, 2007.

[24] C. P. Carter, “An Analysis of the Character Animation in Disney’s Tangled,” Senses Cine., no. 67, 2013.

[25] R. Williams, The Animator's Survival Kit: A Manual of Methods, Principles and Formulas for Classical, Computer, Games, Stop Motion and Internet Animators. Farrar, Straus and Giroux, 2012.

\section{AUTHORS}

Dr Chris Carter is a Senior Lecturer in Animation in the Creative Industries Faculty at Queensland University of Technology. His research focus is on animation aesthetics and animated miseen scène. 